In patients with intrathoracic neoplasms, low forced expiratory volume $\left(\mathrm{FEV}_{1}\right)$ can preclude surgical treatment. Here, we present a case of a giant solitary fibroma of the pleura (SFTP) successfully treated by surgical removal in spite of

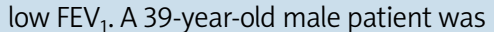
referred to our hospital with dyspnoea and chest distress. Computed tomography $(\mathrm{CT})$ showed a large mass in the left chest. Spirometry showed FEV $1.4 \mathrm{~L}$ (39\% of the expected value). Computed tomography scan-guided transcutaneous aspiration biopsy was performed on the patient, and microscopic examination of the specimen revealed spindle tumor cells with a background of abundant collagen. Complete surgical resection was accomplished. The tumor was large and encapsulated. It measured $28 \mathrm{~cm} \times 20 \mathrm{~cm} \times 18 \mathrm{~cm}$. The definitive diagnosis obtained by histopathology after resection was benign SFTP. The patient felt no dyspnoea at discharge. Surgical treatment of SFTP should be considered even in patients with a huge tumor and with increased post-operative risk.

Key words: dyspnoea, forced expiratory volume, solitary fibroma of the pleura.

\section{A 39-year-old man with dyspnoea, low forced expiratory volume and a large mass of the left hemithorax}

\author{
Zhi-Gang Sun ${ }^{1}$, Xiang-Yan Liu', Zhou Wang ${ }^{1}$, Bao-Zhong Shan'², Min Zhang ${ }^{3}$, \\ Zhe $\mathrm{Li}^{4}$ \\ 1Department of Thoracic Surgery, Provincial Hospital Affiliated to Shandong University, \\ Jinan Center Hospital Affiliated to Shandong University, Jinan, China \\ 2Department of Stomatology, Jinan Center Hospital Affiliated to Shandong University, \\ Jinan, China \\ ${ }^{3}$ Department of Dermatology, Jinan Center Hospital Affiliated to Shandong University, \\ Jinan, China \\ ${ }^{4}$ Department of Pathology, Jinan Center Hospital Affiliated to Shandong University, \\ Jinan, China
}

\section{Introduction}

In patients with intrathoracic malignancies, low forced expiratory volume $\left(\mathrm{FEV}_{1}\right)$ can preclude surgical treatment [1]. However, benign extrapulmonary masses that cause atelectasis may be removable in toto with modest postoperative complications. Here, we present a case of a giant solitary fibroma of the pleura (SFTP) successfully treated by surgical removal in spite of low forced expiratory volume.

\section{Case report}

A 39-year-old male patient was referred to the Provincial Hospital Affiliated to Shandong University with dyspnoea and chest distress. He had a history of smoking. He was found on examination to have decreased breath sounds in the left lower chest, but no other significant clinical findings. Sputum cytology was negative for malignant cells. A chest radiograph (Fig. 1) and computed tomography (CT) scan (Fig. 2 A, B) showed a large mass in the left chest attached to the diaphragm. The density of the mass was lower than the musculature. Spirometry showed: low forced expiratory volume $\left(\mathrm{FEV}_{1}\right)$ 1.4 I (39\% of the expected value); forced vital capacity (FVC) 2.01 (44\%) and maximum voluntary ventilation (MVV) $89 \mathrm{l} / \mathrm{min}$ (56\%). Computed tomography scan-guided transcutaneous aspiration biopsy was performed on the patient, and microscopic examination of the specimen revealed spindle tumor cells with a background of abundant collagen. We performed a left thoracotomy. The tumor was large and encapsulated. It measured $28 \mathrm{~cm} \times 20 \mathrm{~cm} \times 18 \mathrm{~cm}$ (Fig. 3). The mass occupied most of the left and right hemithorax and originated from diaphragmatic parietal pleura. There was an attachment of the tumor to the diaphragm. The patient underwent complete surgical resection of the tumor combined with a partial diaphragm resection. Microscopic examination of the tumor revealed spindle-shaped cells in combination with collagen deposition in a disorderly or random pattern (so-called patternless pattern, Fig. 4A, B) without necrosis and without severe nuclear atypia, and they were rarely mitotic (less than 4 mitoses per high-powered field). Immunohistochemistry demonstrated that the tumor cells stained positive for CD34 and vimentin, and negative for S100 and calretinin. A final diagnosis of benign SFTP was made. The patient felt no dyspnoea at discharge (day 14) and had no local recurrence in annual chest $X$-ray radiographs. The study was approved by the medical ethics committee for human studies of Shandong University of Medical Sciences. 


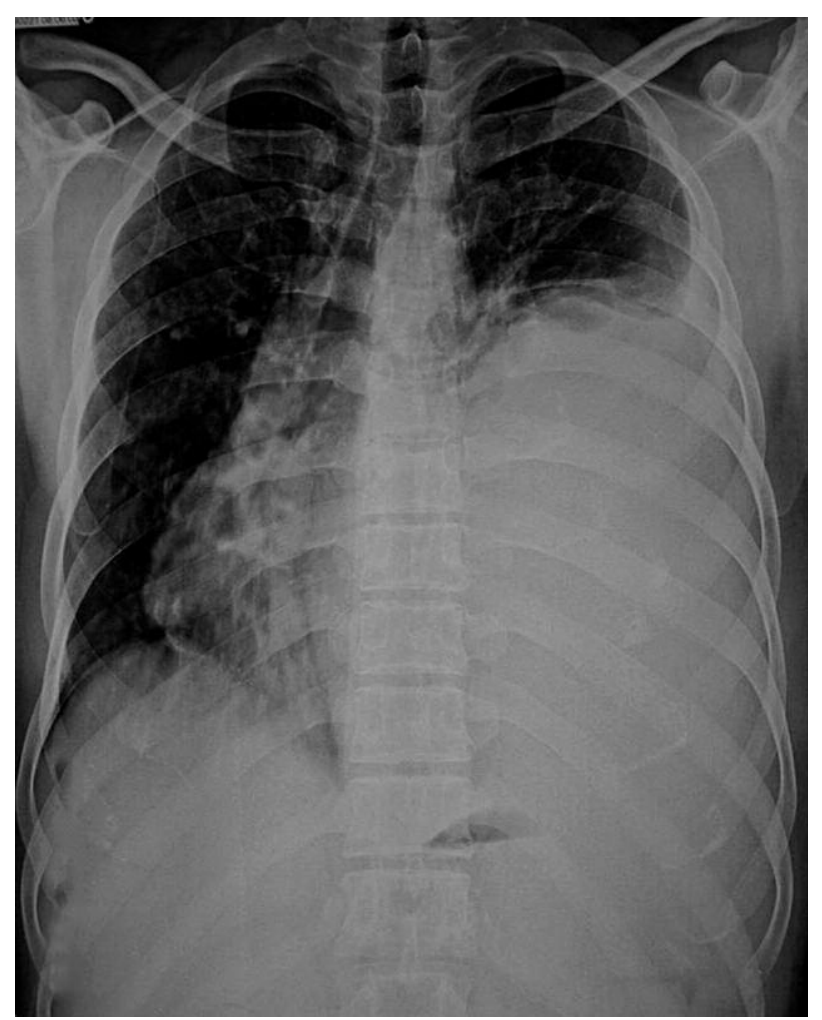

Fig. 1. Chest X-ray showed a large mass in the left hemithorax
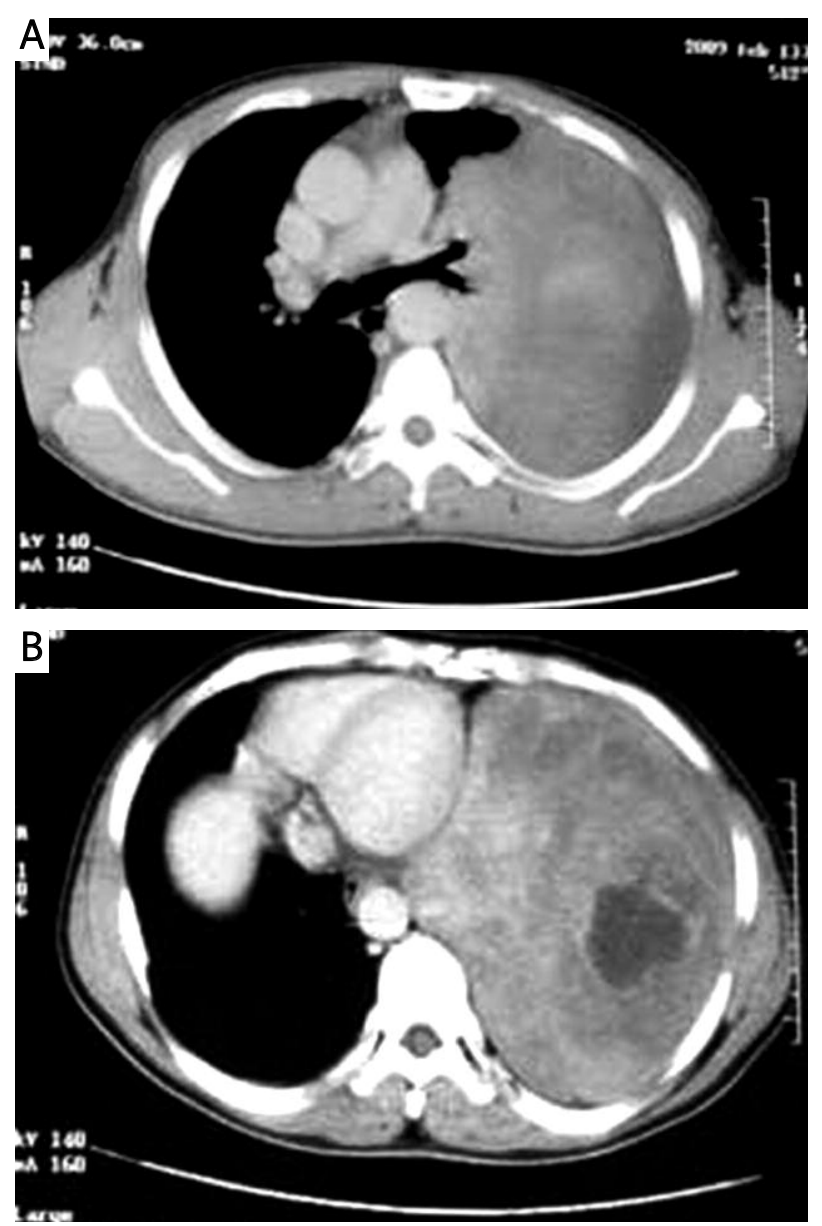

Fig. 2A, B. Computed tomography of the thorax showed a large, solid mass in the left chest attached to the diaphragm

\section{Discussion}

Solitary fibrous tumours of the pleura were first reported in 1870 by Wagner and fewer than 800 cases have been reported in the literature [2]. About half of the patients are asymptomatic and receive diagnoses based on incidental radiographic findings. In cases of larger tumours resulting in compression of the surrounding structures, vague chest pain, chest distress, dyspnoea, or cough may be the main complaint. The larger the tumor, the more likely that symptoms will be present. Hypertrophic pulmonary osteoarthropathy (HPO) is a specific symptom of SFTP, which has been reported in up to $22 \%$ of patients [3]. Significant hypoglycaemia is also related to SFTP in approximately $4 \%$ of cases [4]. In our study, the patient had nonspecific thoracic symptoms caused by the compression.

The usual initial diagnostic test for SFTP is a chest radiograph, which is not specific but serves to document the presence of a mass in the chest. The chest CT scan is the key examination, which more clearly shows the size and location of the tumor and aids in surgical planning. Both the benign and malignant varieties of SFTP usually appear as welldelineated, often lobulated masses that are usually heterogeneous in attenuation [5]. A CT-guided aspiration biopsy was not considered a reliable tool because of its low diagnostic accuracy as reported by several authors [6]. Most authors recommend against needle biopsy in this neoplasm since it does not influence the need for surgical treatment of this obviously resectable mass [5]. Nevertheless, we obtained a satisfactory conclusive diagnosis by biopsy in the case. Appropriate biopsy site, adequate specimens, and reference to immunohistochemical analysis may be of benefit for preoperative pathological diagnosis. Therefore, we proposed that CT-guided core needle biopsy combined with immunohistochemical analysis was a safe and rapid method to provide a confirmatory diagnosis, especially for patients with larger lesions and low forced expiratory volume. Magnetic resonance imaging (MRI) can be a useful alternative, but its use is limited to establishing fibrous tissue characteristics. A benign characteristic can be predicted by low-intensity signals on T2-weighted imaging, in contrast to the high intensity signals found in images of pulmonary carcinoma.

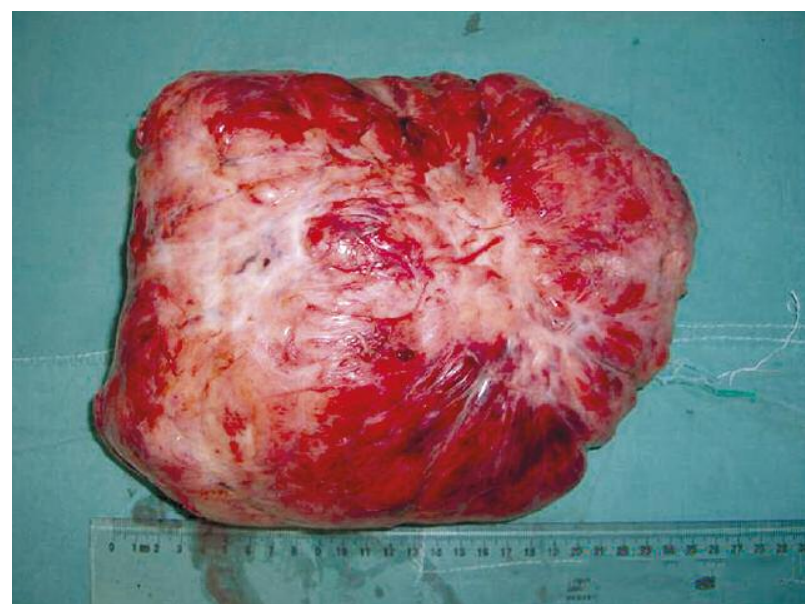

Fig. 3. The operative specimen showed the large dimension of the pleural fibroma 

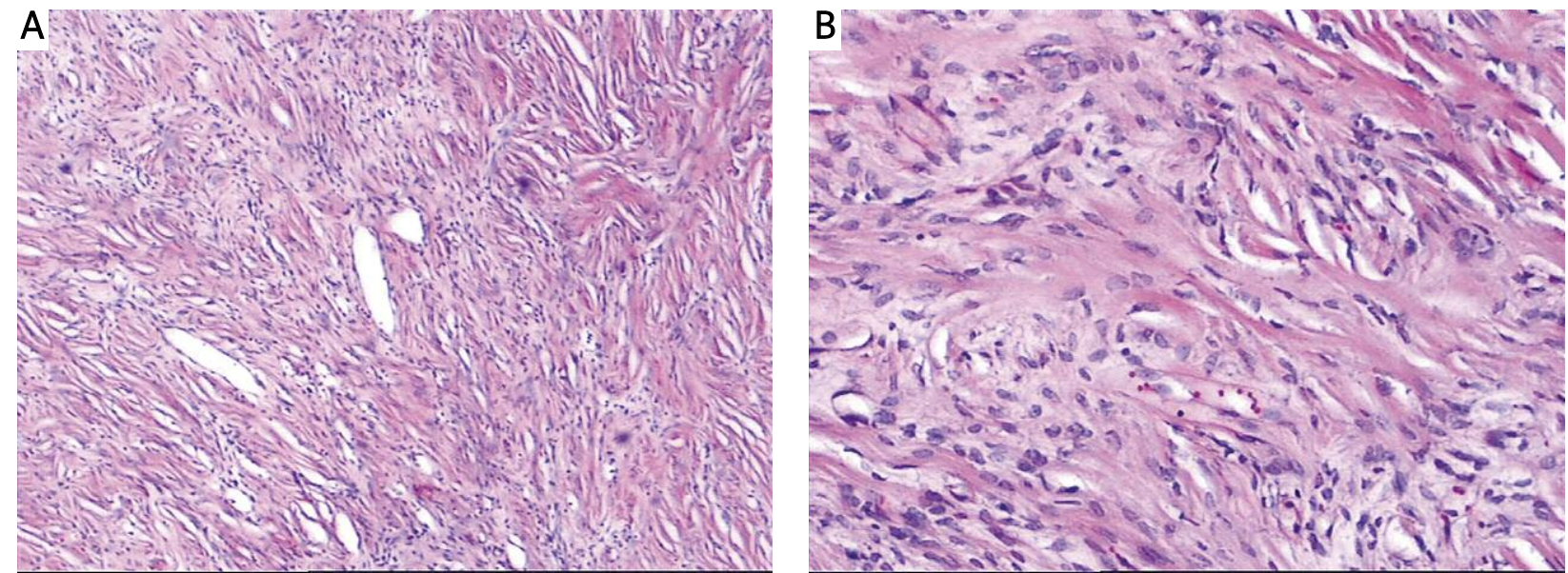

Fig. 4. Results of hematoxylin-eosin staining. Microscopic examination revealed spindle-shaped cells in combination with collagen deposition in a disorderly or random pattern; A) original magnification 40x and B) original magnification 100x

The role of positron emission tomography (PET) scanning in the imaging of pleural disease is currently being debated and more experience is needed before conclusions can be reached [2].

Spirometry is a simple, inexpensive, standardized, and readily available test. Spirometric indexes are commonly tested and have been extensively studied, including $\mathrm{FEV}_{1}$, $\mathrm{FVC}$, and MVV. Of all these indexes, $\mathrm{FEV}_{1}$ is regarded as being the best for predicting complications of malignant intrapulmonary tumour resection in the initial assessment, and $\mathrm{FEV}_{1}<40 \%$ of the predicted value usually signals that surgery should be deferred [1]. However, in this case, the mass was extrapulmonary, non-invasive and benign, and the diagnosis warranted that complete removal was possible [7]. The patient had neither cardiovascular nor parenchymal pulmonary disease, but dyspnoea and chest distress disabled the patient in all daily activities. In this case the increased perioperative risk was clearly outweighed by the inevitable progression of disease. Presently, there is no firm evidence in the literature that supports that patients with SFTP could benefit from radiotherapy or chemotherapy and the best treatment of giant SFTP is complete surgical resection. It is a challenging task because of poor exposure, significant blood supply, and especially low forced expiratory volume. A giant sessile tumor with good movement may compress the heart and draw down blood pressure when the thoracic cavity is open. The $45^{\circ}$ lateral decubitus position during the operation is an easy and reasonable way to avoid compression. Peri-tumor extensive adhesions make it more difficult to ligate feeding vessels and handle hilar vessels, especially in a tumor with a broad-based pedicle. Piecemeal resection proved to be a practical method for removal of giant SFTP, because it can achieve better exposure and avoid impairing adjacent structures. Extended excision is necessary in cases of invasive tumor. Atelectasis occurs frequently in giant SFTP, and removal of a giant tumor might result in pulmonary oedema. It is beneficial to use mechanism ventilation with positive end-expiratory pressure (PEEP) during the first postoperative day. And it is important for these patients to undergo chest $\mathrm{X}$-rays to check postoperative lung reexpansion.
In conclusion, surgical treatment of SFTP should be considered even in patients with a huge tumor and with increased post-operative risk.

The authors declare no conflict of interest.

The authors would like to thank Shan Dong University for its cooperation.

\section{References}

1. Kaza AK, Mitchell JD. Preoperative pulmonary evaluation of the thoracic surgical patient. Thorac Surg Clin 2005; 15: 297-304.

2. Patel N, Bishay A, Bakry M, George L, Saleh A. Dyspnea with slowgrowing mass of the left hemithorax. Chest 2007; 131: 904-8.

3. Robinson LA. Solitary fibrous tumor of the pleura. Cancer 2006; 13: 264-9.

4. Filosso PL, Asioli S, Ruffini E, et al. Radical resection of a giant, invasive and symptomatic malignant Solitary Fibrous Tumour (SFT) of the pleura.Lung Cancer 2009; 64: 117-20.

5. Mitchell JD. Solitary fibrous tumor of the pleura. Semin Thorac Cardiovasc Surg 2003; 15: 305-9.

6. Harrison-Phipps KM, Nichols FC, Schleck CD, et al. Solitary fibrous tumors of the pleura: results of surgical treatment and long-term prognosis. J Thorac Cardiovasc Surg 2009; 138: 19-25.

7. Sung SH, Chang JW, Kim J, Lee KS, Han J, Park SI. Solitary fibrous tumors of the pleura: surgical outcome and clinical course. Ann Thorac Surg 2005; 79: 303-7.

\section{Address for correspondence}

\section{Zhou Wang MD}

Department of Thoracic Surgery

Provincial Hospital Affiliated to Shandong University

Jinan 250021, China

tel. (0086)13665312567

e-mail:wangzhouszg@126.com

$\begin{array}{ll}\text { Submitted: } & 23.12 .2011 \\ \text { Accepted: } & 15.02 .2012\end{array}$ 